\title{
Research and Development of Portable Thermoelectric Generator Using Peltier Plates and Waste Heat ${ }^{\dagger}$
}

\author{
Mohammad Monirujjaman Khan ${ }^{1}$ \\ ${ }^{1}$ Department of Electrical and Computer Engineering, North South University, Bashundhara, Dhaka-1229 \\ * Correspondence: monirujjaman.khan@northsouth.edu; Tel.: +8801779006296 \\ + Presented at the 1st International Electronic Conference on Applied Sciences \\ 10-30 November 2020; Available online: 10/11/2020 -30/11/2020
}

Published: 10 November 2020

\begin{abstract}
There is a growing demand for alternative sources of energy in the current world and extensive research work is being done to find out ways to do so as well as increase the efficiency of the existing methods. Thermoelectric power generation has emerged as a promising green technology due to its many advantages. Thermoelectric generators use thermoelectric modules, a solid state device, which can convert thermal energy to electrical energy from a temperature gradient and works by using the Seebeck effect. These can be utilized to harness waste heat that is abundant in homes and industries. In rural or remote areas, where electricity from the national grid is not available, thermoelectric generators can be used to power electronic devices. This paper demonstrates the potential of thermoelectric power generation using Peltier plates and how it can be used to harness waste heat using a portable thermoelectric generator. The proposed system has been developed and tested. It can generate power which is useful for low voltage electric devices. The device is portable, cheap and user-friendly.
\end{abstract}

Keywords: Power; Electricity; Thermoelectric Generator; Peltier Plates; Seebeck Effect; Boost Converter; Waste Heat; DC-DC Boost Converter.

\section{Introduction}

Electricity is one of the most essential elements of our daily life. It is used everywhere in our life. Electricity is a scarce resource and the demand for electricity is always increasing. Conventional energy generation plants use non-renewable sources of energy like gas and coal. With increased interest and existing limitations, a requirement arises for alternative sources of energy in the present world and a wide research effort is being performed to realize the ways to increase the efficiency of the existing methods. In Bangladesh load shedding is a very common problem. There nearly $64 \%$ people are living in the rural areas of our country [1]. Due to lack of electrical energy load shedding is occurring on a regular basis and it is the most in the rural areas. During the hot season the load shedding is the most in areas. In some remote rural areas in our country electricity is not still available. More than a quarter of the rural population still lacks access to electricity and relies on fuel wood and kerosene for energy [2]. People need to suffer a lot due to lack of electricity in the rural areas. Table 1 shows the amount of power generation in Bangladesh from each source. The power distribution system is lagging behind the ever-growing customer base, resulting in daily load shedding across the country. It is also common in city areas including the capital of Bangladesh (Dhaka). Official data show the distribution system has not grown in synchronization with growth in demand [3]. 
Nowadays the crisis of energy has become a most important challenge in our country. Figure 1 (a) shows the scenarios when load shedding occurred in Bangladesh. It is very urgent to find alternative energy sources to solve the national big issues. In our country, solar based alternative energy producing systems are being used nowadays but this requires the sunlight to produce energy. Application of the solar system in the house of remote rural areas in Bangladesh is shown in Figure 1(b). Solar system is sunlight dependent. Therefore more alternative energy harvesting techniques are very important nowadays from nature to fulfill the demand of national electrical energy demand for our country.

Recently, research interest is growing in this area to find alternative energy sources. Currently research is also carried out worldwide on renewable energy sources while trying to make the current systems such as solar more efficient [4]. Design of solar-panel energy systems is presented in open literature [5]. The PowerPot is a thermoelectric generator that uses heat to generate electricity is presented in [6]. This product has been developed abroad and it is costly to bring in Bangladesh. It will be hard for the poor people to afford it. In this paper a portable thermoelectric generator system has been developed using Peltier plates and waste heat. The system can produce electricity from the waste heat. Thermoelectric generators are mostly used to convert the waste heat so it is considered as a green technology. The developed system is cheap, portable, affordable and compact. It uses the seed Seebeck effect technique [6]. The system is very user friendly and it costs only 39 US Dollar which is equivalent to 3300 Bangladeshi Taka.

Table 1. Amount of power generation in Bangladesh of each source.

\begin{tabular}{ccc}
\hline Unit Type & Capacity (unit) $\mathbf{M W}$ & Total (\%) \\
\hline Coal & 2500.00 & 2.39 \\
\hline Gas & 6719.00 & 64.33 \\
\hline HFO & 1963.00 & 18.79 \\
\hline HSD & 783.00 & 7.5 \\
\hline Hydro & 230.00 & 2.2 \\
\hline Imported & 500.00 & 4.79. \\
\hline Total & 10445.00 & 100 \\
\hline
\end{tabular}

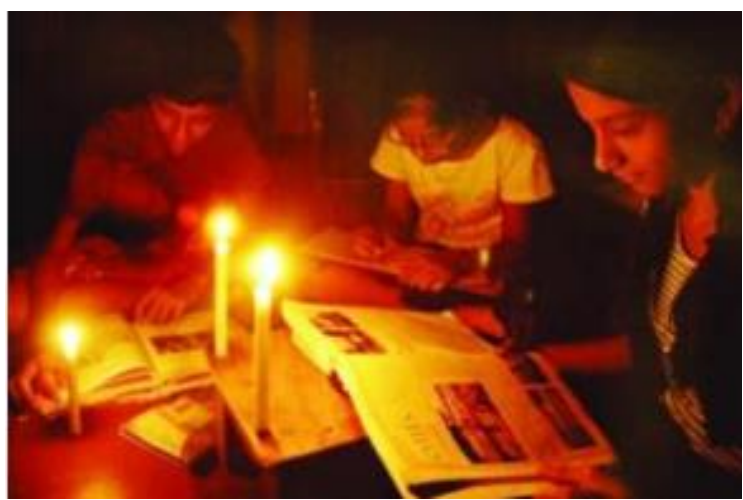

(a)

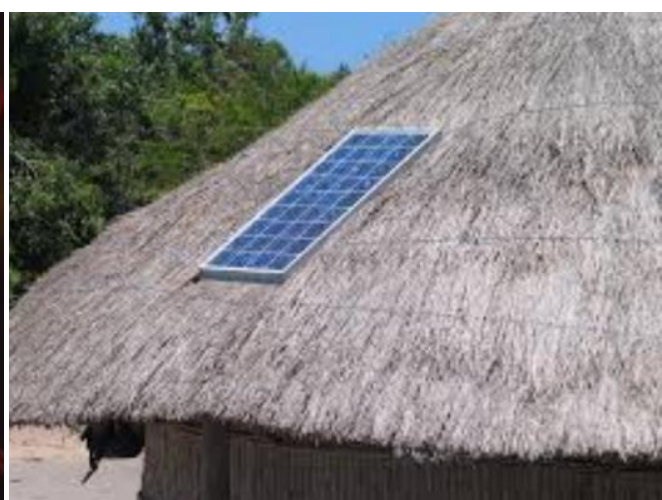

(b)

Figure 1. (a) Scenarios when load shedding occurred [3], (b) Application of solar system in the house of remote rural areas in Bangladesh [2].

\section{Methodology}

Figure 1 shows the block diagram of the thermoelectric generator using Peltier plates and waste heat. This system is designed through the use of hardware components. The main required hardware components are DC-DC Boost Converter XL 6009, Navo 5 volts Voltage Regulator, Thermoelectric Module-TEC 12706 Peltier module, Volts Rechargeable Lead-Acid Battery, connecting wires, Aluminum Water Reservoir, Steel Plates, Thermal Paste. Table 2 lists the required 
hardware components and the cost of the hardware components in BD Taka and USD. The total cost of the system is 39 US Dollar which is equivalent to 3300 Bangladeshi Taka.

A thermoelectric power generator is a solid state device which can directly convert heat to electricity using the Seebeck effect. The Seebeck effect is a phenomenon in which a temperature difference between two dissimilar electrical conductors or semiconductors produces a voltage difference between the two substances. Thermoelectric generators (TEGs) produce a current flow in an external circuit by the imposition of a temperature difference $\Delta \mathrm{T}$ across the TEG. The magnitude of this $\Delta \mathrm{T}$ determines the magnitude of the voltage difference $\Delta \mathrm{V}$ and the direction of heat flow determines the voltage polarity. The magnitude of the open-circuit TEG voltage is determined by the Seebeck coefficient and the magnitude of the absolute temperature [6]. In this system Thermoelectric -TEC 12706 Peltier module has been used; see Fig. 3 (b) [7]. Its work is characterized by side refrigeration and side fever. This TEG module is of great usefulness and it is heated one side on the other hand the other side is cooled using a coolant to create a temperature difference and thus a potential difference across the plates. One side of the TEG is connected to the steel plate and the other side is connected to the cool side using thermal paste. The resultant voltage is attached to a DC-DC boost converter to improve the voltage to $6 \mathrm{~V}$ and start the charging method of the battery. Figure 4 (a) shows DC-DC Boost Converter XL 6009 that is used in this system. The XL6009 module is a DC to DC BUCK-BOOST converter module that operates at a switching frequency of $400 \mathrm{kHz}$ [8].Volts rechargeable lead-acid $6 \mathrm{~V}$ battery has been used to harvest the energy from the system; see Figure 3 (a) [9]. The energy produced from the system can be stored in this battery. A Navo 5 volts voltage regulator is used in this system that is attached to the output of the battery to set the voltage of the system to the preferred output necessity. Figure 4 (b) shoes the Navo 5 volts voltage regulator [10]. A voltage regulator is a system designed to automatically maintain a constant voltage level. A voltage regulator may use a simple feed-forward design or may include negative feedback. It may use an electromechanical mechanism, or electronic components.

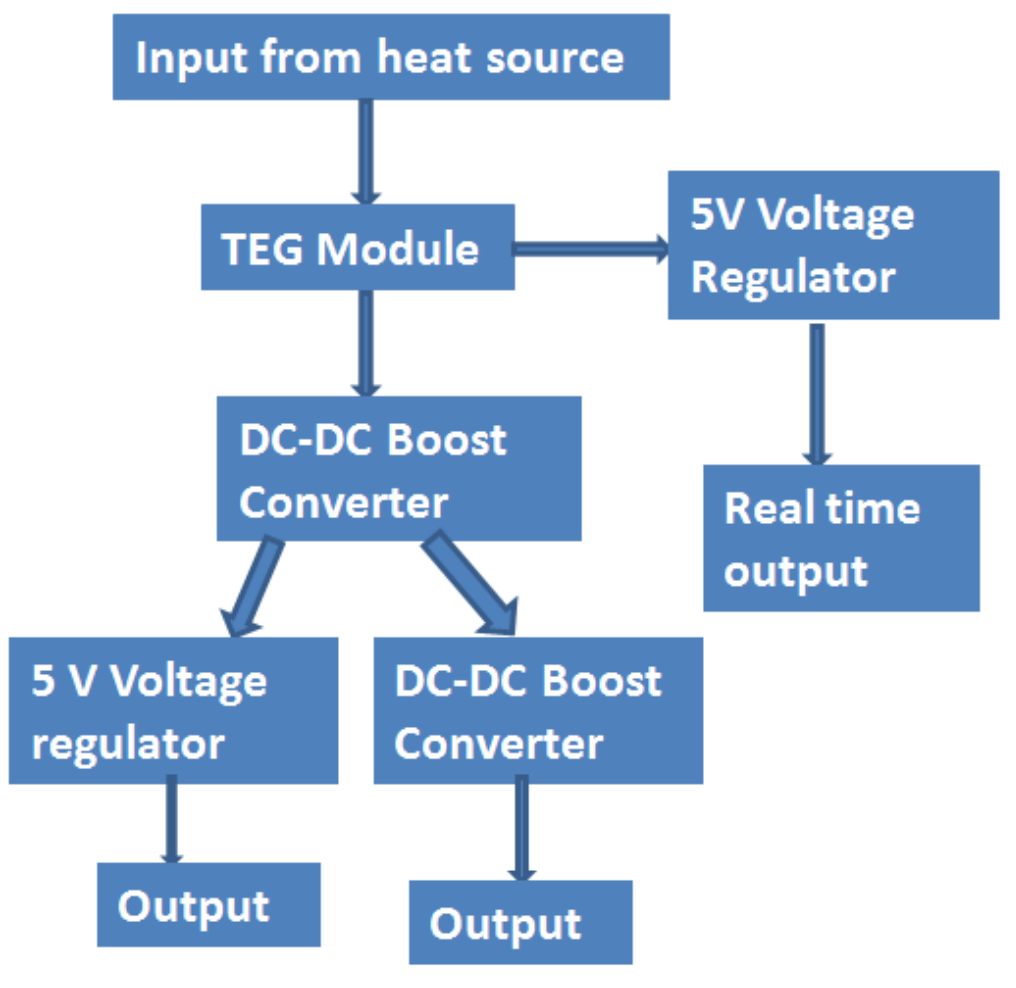

Figure 2. Block diagram of the whole system of the thermoelectric generator using Peltier plates and waste heat.

Table 2. List of required hardware and cost of the hardware components. 


\begin{tabular}{cccc}
\hline No & Name of the Components & $\begin{array}{c}\text { Price in BD } \\
\text { Taka }\end{array}$ & Price in USD \\
\hline 1 & DC-DC Boost Converter XL 6009 & 350 & 4.13 \\
\hline 2 & Navo 5 volts Voltage Regulator & 100 & 1.18 \\
\hline 3 & TEC 12706 Peltier module & 1150 & 13.56 \\
\hline 4 & Volts Rechargeable Lead-Acid & 600 & 7.08 \\
\hline 5 & Battery & 700 & 8.26 \\
\hline 6 & Aluminum Water Reservoir & 150 & 1.77 \\
\hline 7 & Steel Plates & 150 & 1.77 \\
\hline 8 & Thermal Paste & 100 & 1.18 \\
\hline & Connecting wires & 3300 & 39.00 \\
\hline
\end{tabular}

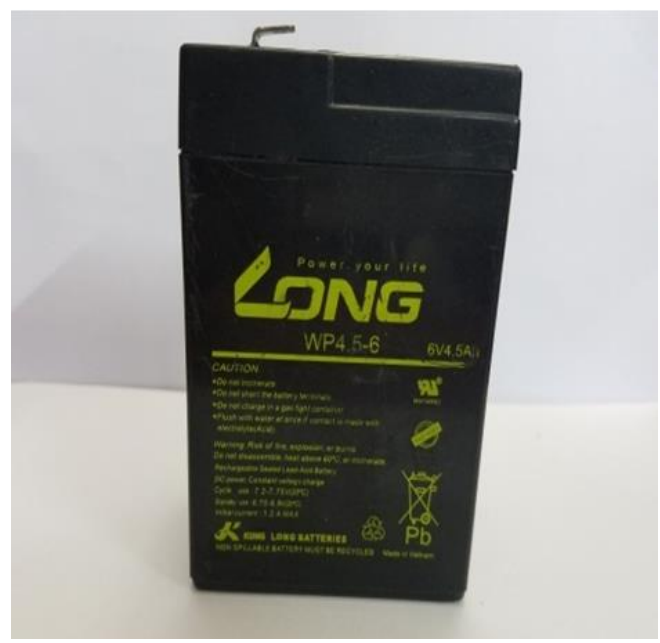

(a)

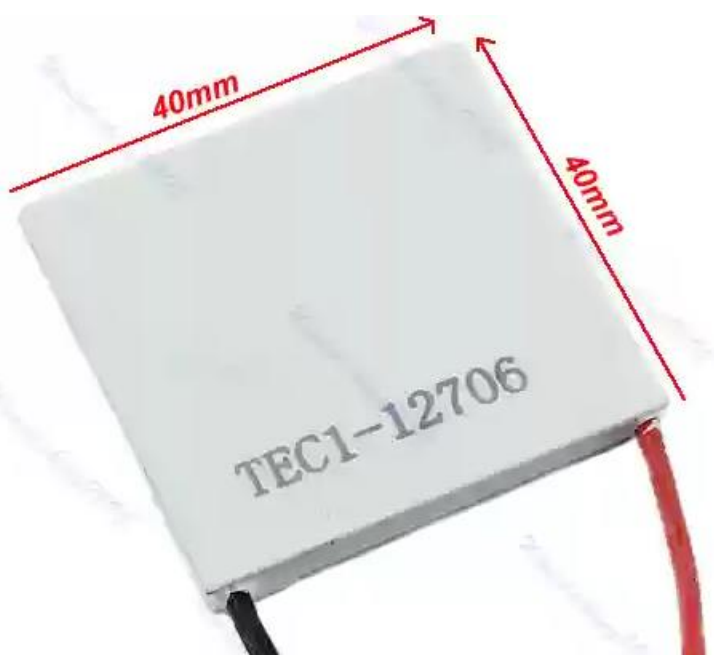

(b)

Figure 3. (a) Volts Rechargeable Lead-Acid Battery [9], and (b) Thermoelectric TEC 12706 Peltier module [7].

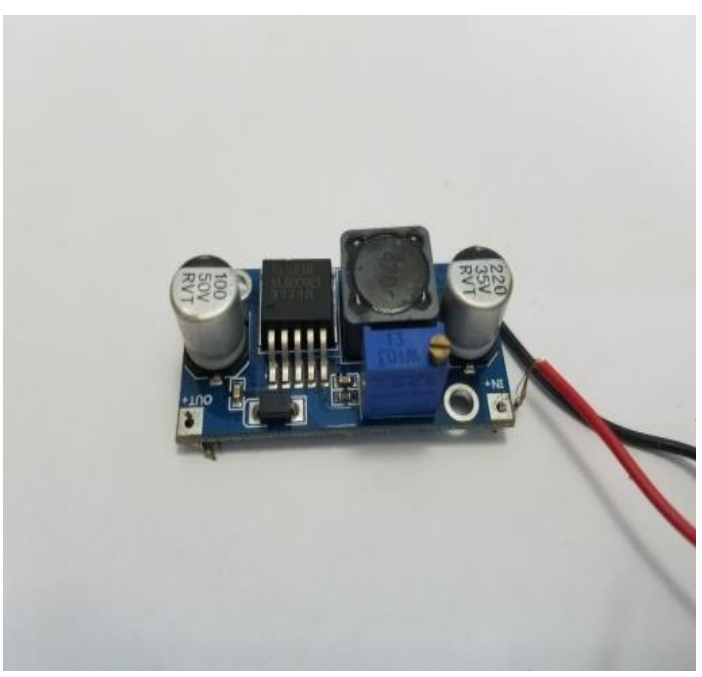

(a)

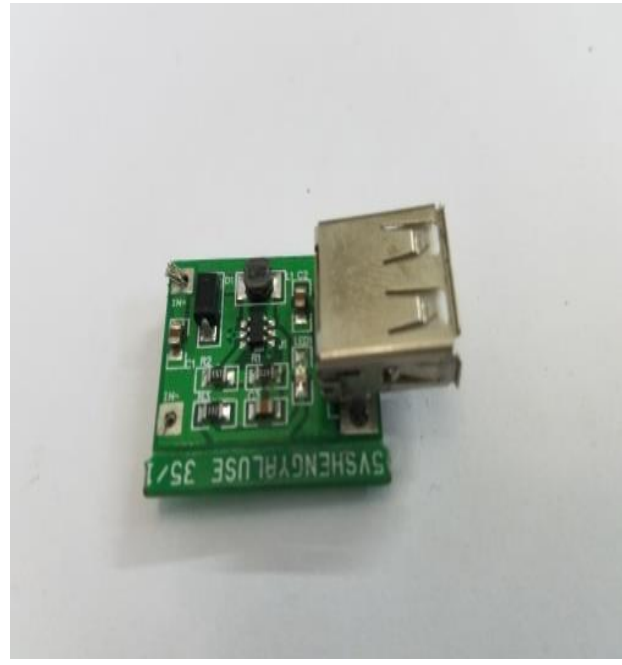

(b)

Figure 4. (a) DC-DC Boost Converter XL 6009 [8] and (b) Navo 5 volts Voltage Regulator [10].

\section{Product System Design and Analysis}


The final thermoelectric system using Peltier plates has been developed. Figure 5 shows the proposed complete system design of the thermoelectric generator using Peltier plates. In the final design steel plates have been used for the hot side (extended steel plate); see Figure 5 . The waste heat is actually applied to the hot side of the steel plate of the TEG system. This extended still plate in the final prototype is actually placed near the gas stove or other places of waste heat. The whole system as a prototype has been tested. Figure 6 presents the complete system design (prototype) of the thermoelectric generator in testing condition. Here in this paper two candles are used to produce the heat and they were placed near the extended steel plates. In this design steel plates have been used due to a high thermal conductivity and low cost but we can also use the other conductive materials. In the next design we will study other materials to see the performance improvement effects. Author is doing further research for this study. An aluminum chamber is used for the cold side which can store water as can be seen in Figure 5. This steel plate is attached with the aluminum chamber. The surface of the aluminum storage chamber is connected to the cool surface of the module with thermal paste. The module used in this system transmits the heat to the aluminum chamber followed by water via conduction. After that in this way the water soaked up the heat and improved the temperature variation, as a result amplifying the output voltage. This system has integrated two outputs with it. These two outputs are connected in parallel. Out of these two outputs one is attached to the 5 volts voltage regulator. In real life scenarios when the system is placed near the waste heat it can charge the mobile charger or other low voltage devices from this output. Remaining output part is attached with a booster module which increases the voltages to 8 Volts therefore the battery can be charged.

Thermoelectric power is the conversion of a temperature differential directly into electrical power. It follows the Seebeck effect as mentioned earlier. Seebeck noticed that when a loop composed of two dissimilar materials was heated on one side, an electromagnetic field was created. He noted that the strength of the electromagnetic field, and therefore the voltage, is proportional to the temperature difference between the hot and cold sides of the material.

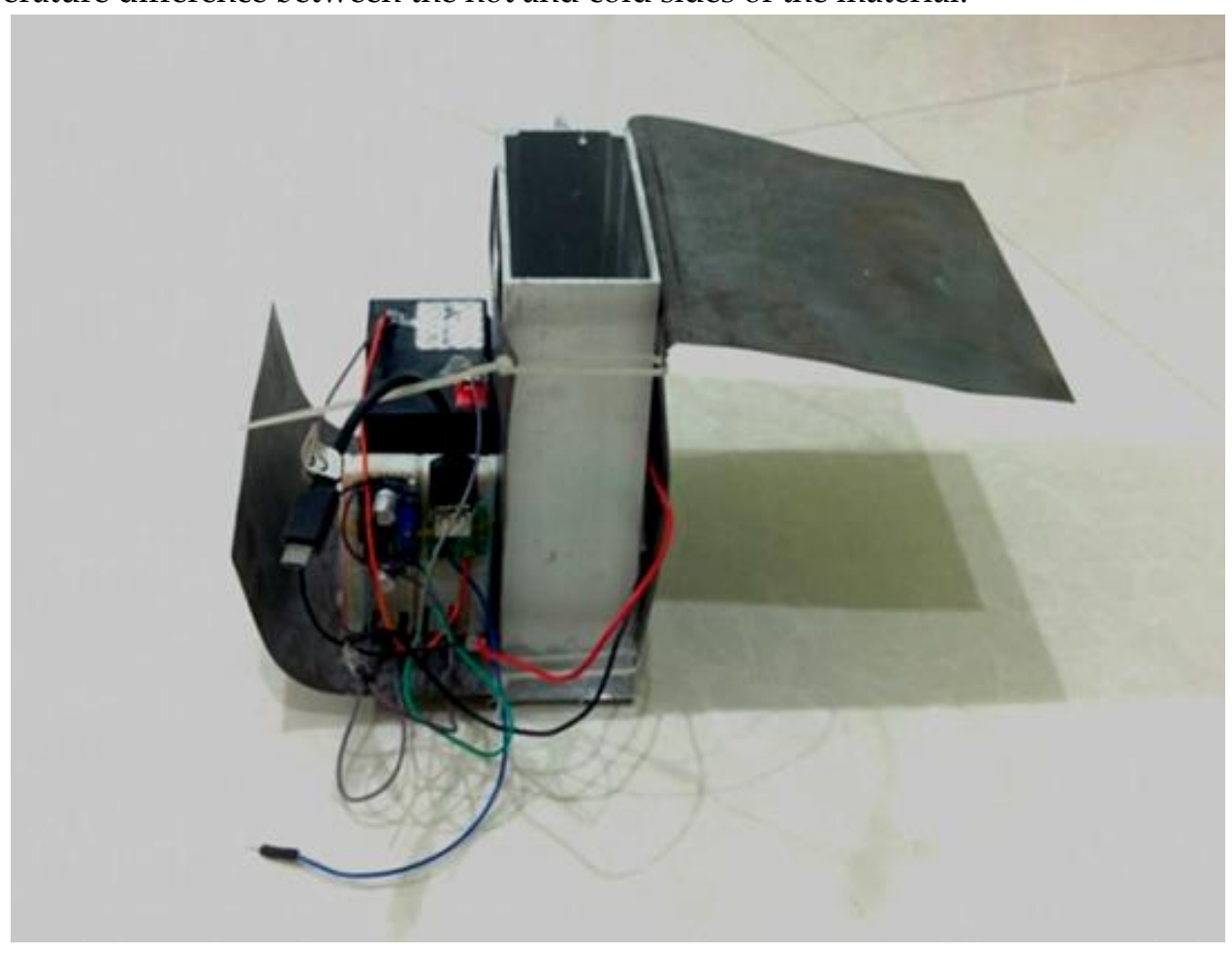

Figure 5. Proposed complete system design (prototype) of the thermoelectric generator using Peltier plates.

In this study, the output voltage has been measured and from the results it is noticed that the voltage increases very quickly for the first period of time, like the charging of a capacitor. The rate decreases as time passes. This is due to the decreases of temperature difference because the cold side 
cannot cool as speedy as the hot side heats up. Maximum of 2.7 output voltage is obtained from this system. In this system the Navo voltage regulator is capable of stepping up voltages above $1 \mathrm{~V}$ to give a constant out of $5 \mathrm{~V}$. For charging a cell phone minimum 5 volt is essential therefore we have used this voltage regulator. This system when measured maximum of 300-400 mA output current is noticed. We have tested this system in real life and charged a mobile phone. Using this system in real life application we have powered up a USB fan and LED bulb. We were also able to store the energy in a battery. due to lack of space limitation more technical analysis and design and testing results are not possible in this paper. However the author of this paper is working further to improve the efficiency and with the results an article will be submitted in MDPI for review.

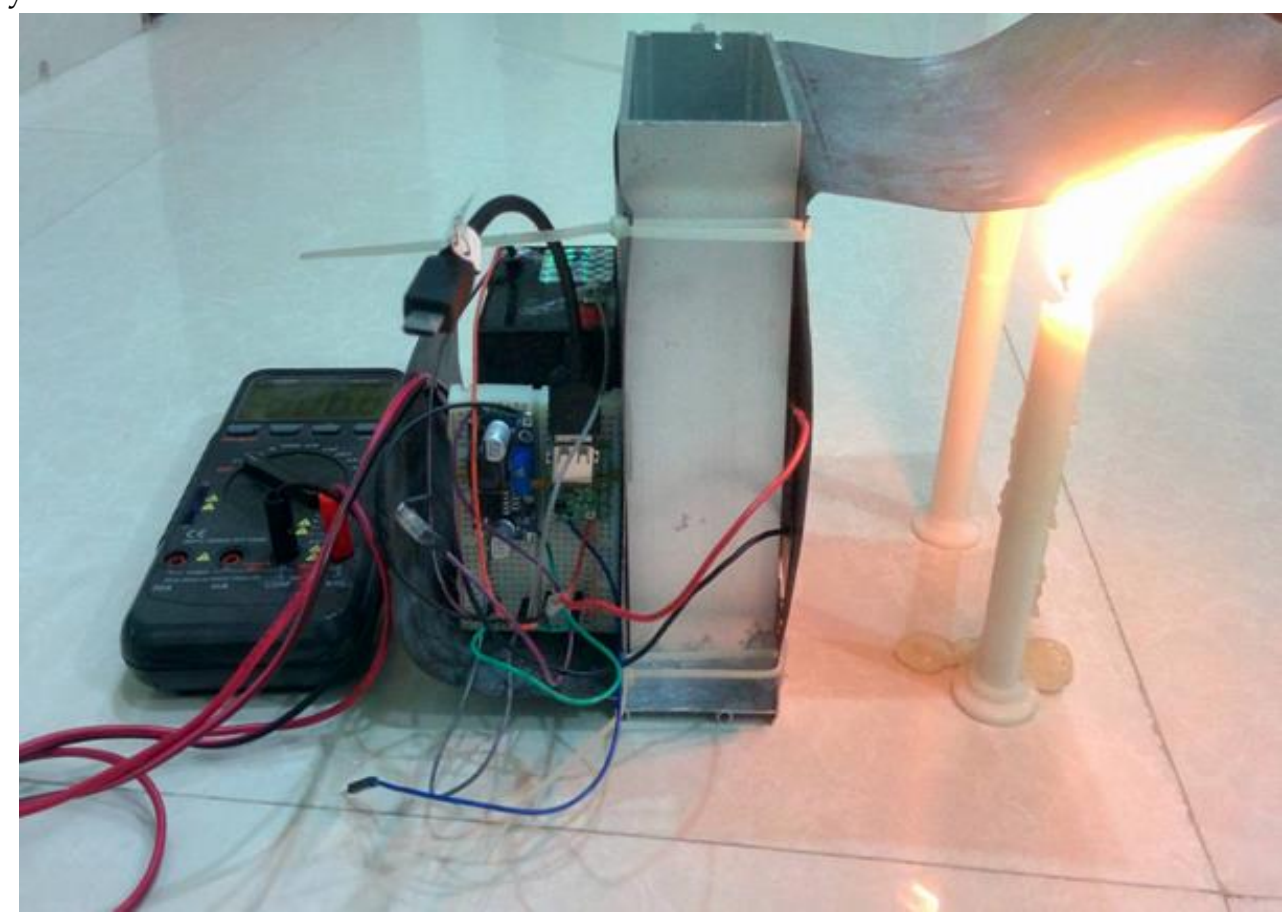

Figure 6. Complete system design (prototype) of the thermoelectric generator using Peltier plates.is in testing condition. .

\section{Conclusions}

Research and development of portable thermoelectric generators using Peltier plates and Waste heat are presented in this paper. The system has been designed as a prototype and it has been tested in real life scenarios. It shows very good performance. The author is now working further to improve the performance of the system. The proposed developed prototype is low cost, user friendly, portable, green technology, capable of generating electricity, able to store produced energy and compact. Due to its low cost it will be affordable and mass rural people in Bangladesh can buy this system. Rural people can use this system during cooking and store energy or they can use the produced energy for real time mobile charging and other devices. Due to load shedding this device has huge demand in our country which will make this system sustainable. Life of rural people will be comfortable with the use of this system. This system can be also scaled up for the uses of industrial areas. This is an emerging technology with huge potential. This system can transform the power sector of Bangladesh. In future using more powerful TEG modules can improve the performance of the system. It can also help to improve the efficiency using different conductive materials, cooling systems and more TEG modules.

Author Contributions: Author contributed for the development of the system. The author of the paper also has written the manuscript. Currently research and development are going on for the improvement of the efficiency of the existing system. The new work has been carried out by other researchers with the supervision and guidance of the author of this paper. 
Funding: This research received no external funding.

Acknowledgments: Author of this paper would like to thank the Department of Electrical and Computer Engineering of North South University. The development and integration work of this paper was carried in the Engineering Laboratory in the Department of Electrical and Computer Engineering at North South University.

Conflicts of Interest: The authors declare no conflict of interest.

\section{References}

1. Share of rural population Bangladesh 2009-2018. Available online: https://www.statista.com/statistics/760934/bangladesh-share-of-rural-population/\#: :text=In\%202018\%2C \%20approximately\%2063.37\%20percent,in\%20rural\%20areas\%20in\%202009. (accessed on 10th October 2020 ).

2. Load shedding even after having double capacity. Available online: http://www.dailyindustry.news/load-shedding-even-double-capacity/(accessed on 10th July 2020 ).

3. Solar power a one stop-solution in rural Bangladesh. Available online: https://www.responsiblebusiness.com/news/asia-pacific-news/solar-power-a-one-stop-solution-in-rural-b angladesh/(accessed on 10th July 2020 ).

4. 11 Different sources of alternative energy. Available online: https://ecavo.com/alternative-energy-sources/ (accessed on 10th July 2020 ).

5. F. L. Luo, "Design of solar-panel energy system," 2011 6th IEEE Conference on Industrial Electronics and Applications, Beijing, 2011, pp. 2304-2309,

6. How do thermoelectric works? Available online: https://powerpractical.com/pages/how-do-thermoelectrics-work (accessed on 10th July 2020 ).

7. Thermoelectric TEC 12706 Peltier module. Available online: https://leetechbd.com/product/tec1-12706-thermoelectric-cooler-peltier/(accessed on 11th July 2020 ).

8. XL6009 DC-DC Buck Boost Converter Module. Available online: https://components101.com/modules/xl6009-dc-dc-step-down-voltage-regulator-module (accessed on 11th July 2020 ).

9. Volts rechargeable lead-acid $6 \mathrm{~V}$ battery. Available online: https://www.amazon.com/Volt-Rechargeable-Sealed-Lead-Battery/dp/B00BSEJYCM(accessed on 11th July 2020 ).

10. Navo DC 1 5V USB Output Voltage Boost Regulator Module - Green. Available online: https://www.dx.com/p/navo-dc-1-5v-usb-output-voltage-boost-regulator-module-green-2035782.html\#.X 8MBXWX7SUk (accessed on 15th July 2020 ). 